\title{
Building Semantic/Ontological Knowledge by Text Mining
}

\author{
Eduard Hovy \\ Information Sciences Institute \\ of the University of Southern California \\ 4676 Admiralty Way \\ Marina del Rey, CA 90292-6695 \\ U.S.A. \\ email: hovy@isi.edu \\ http://www.isi.edu/natural-language/nlp-at-isi.html
}

\begin{abstract}
People have long talked about having NLP systems employ semantic knowledge on a large scale. However, no-one has yet built a large ontology that was indeed practically useful for tasks such as question answering, machine translation, and information retrieval. Work on WordNet, a major contender, shows that it requires more content to realize its full potential, while efforts to use CYC show how hard it is to build general-purpose ontologies that can support NLP applications. In this talk I outline some recent efforts to automatically acquire knowledge that may be placed into terminological ontologies and used by NLP systems, and mention some problems in evaluating the quality of the results.
\end{abstract}

